\title{
Microwave Doppler Radar for Heartbeat Detection Vs Electrocardiogram
}

\author{
D. Obeid, G. Zaharia, Member, IEEE, S. Sadek, and G. El Zein
}

\begin{abstract}
This paper presents a contact-less system to detect the heartbeat activity from a distance of one meter. The proposed system is based on using a vector network analyzer. Measurements are performed at $16 \mathrm{GHz}$ for different power levels between 0 and $\mathbf{- 2 5} \mathbf{~ d B m}$. Results for two S-parameters are obtained and compared to an ECG simultaneous signal in terms of heartbeat rate and heart rate variability.
\end{abstract}

Index Terms-Doppler effect, heart rate variability, remote monitoring, VNA.

\section{INTRODUCTION}

' 'HE utility of microwave Doppler radar has increased for several applications. These include home health care applications, urgent conditions, and hospital needs. Affixed electrodes for traditional electrocardiograms are perturbing for long-duration home monitoring, as well as for patients with conditions such as burn victims or newly born infants. In addition, a touchless technique is necessary to detect life signs for people under rubble [1].

Based on the Doppler theory, a target with a quasi-periodic movement reflects the transmitted signal with its phase modulated by the time-varying position of the target [2]. When the target is the person's chest, the reflected signal contains information about the chest displacement due to heartbeat and respiration. However, while holding breath, the reflected signal depends on the chest displacement due to heartbeat alone. At rest, the variation of the chest displacement, caused by respiration, is between 4 and 12 $\mathrm{mm}$ [3], and the chest displacement due to heartbeat alone ranges between 0.2 and $0.5 \mathrm{~mm}$ [4]. The respiration rate corresponds to a frequency that varies between 0.1 and 0.3 $\mathrm{Hz}$, while the heartbeat rate (HR) corresponds to a frequency that varies between 0.83 and $2 \mathrm{~Hz}[5]$.

Previous works tend to detect life signs $[6,7]$, respiration rates and heartbeat rates, using fixed frequency and fixed power of the transmitted signal. Direct-conversion Doppler radars, operating at $1.6 \mathrm{GHz}$ and $2.4 \mathrm{GHz}$, have been integrated in $0.25 \mu \mathrm{m}$ CMOS and BiCMOS technologies [8]. Heart and respiration activities were detected using a modified Wireless Local Area Network PCMCIA card, and

The authors acknowledge CEDRE (project reference number: 08SciF6/L4), AUF (project reference number: G3-110/1649), and "Région Bretagne" for partially funding this work. a module combining the transmitted and reflected signals [9]. Other systems operating in the Ka-Band were described in [10] using a low power double-sideband transmission signal. Similarly, quadrature demodulation architecture is used to detect vital signs from distance [11]. A new study showing the possibility of detecting the presence of a person through a wall using Ultra-Wideband (UWB) radar is presented in [12]. Some experiments are performed for the detection of life signs using the $4-7$ $\mathrm{GHz}$ band with $1 \mathrm{~mW}$ power and around $7 \mathrm{~dB}$ antenna gain [13]. A recent study describes a system operating at 94 $\mathrm{GHz}$ that is able to detect the heart and the respiration activities of stationary subjects [14]. Another related published work showed the ability of measuring the heartbeat rate using a Vector Network Analyzer (VNA) and only one antenna [15].

In this paper, a microwave system for heartbeat detection is proposed. The system is based on using a VNA and one (or two) antenna. The aim of this system is to provide a comparative approach in terms of the operational frequency and the transmitted power. This helps identifying the optimum frequency and signal processing technique, for the minimal transmitted power. This study is necessary prior the implementation process. The proposed system, tested for one frequency and several transmitted powers, is validated with an electrocardiogram (ECG) signal.

In addition to the installation simplicity, the proposed system has the ability of tuning both frequency and power. Using a VNA, two measurement techniques are compared using respectively one and two antennas. As the transmission of a signal with minimum power would be safer for both patient and medical staff, measurements are performed for several transmitted power levels between 0 and $-25 \mathrm{dBm}$. Both heartbeat rate and Heart Rate Variability (HRV) are extracted and compared to the values obtained by the ECG. The rest of the paper is organized as follows: Section II presents background information, the proposed system with its two versions, and a smoothing technique. Section III presents the heartbeat signals obtained using two versions of the proposed system: one and two antennas respectively. Section IV states the results of extracting the heartbeat rate and the heart rate variability from both phases of $S_{11}$ and $S_{21}$. Section V concludes the work. 


\section{MEASUREMENT SPECIFICATIONS AND SMOOTHING TECHNIQUE}

This section presents background information about the Doppler radar principle, the proposed system, and a smoothing technique. It is noteworthy to mention that our previous work has demonstrated a feasibility study in the absence of an ECG signal [16, 17]. In this work, measurements were performed simultaneously with an ECG reference signal. In addition, a recent work shows the possibility of detecting the heartbeat signal using a VNA and one antenna $\left(\mathrm{S}_{11}\right)$ [15]. This study provides a comparison between using single antenna $\left(S_{11}\right)$ and two antennas $\left(\mathrm{S}_{21}\right)$.

\section{Background Information}

The chest displacement varies between $4 \mathrm{~mm}$ and 12 $\mathrm{mm}$ due to respiration, while it ranges between $0.2 \mathrm{~mm}$ and $0.5 \mathrm{~mm}$ due to heart beatings. The measurement of this small displacement is the objective of this work. The proposed system detects the heartbeat activity upon the detection of the phase variation of parameters $S_{11}$ or $S_{21}$ measured by the VNA. The phase variation is directly proportional to the chest displacement and indirectly proportional to the wavelength of the transmitted signal. The relation between the chest displacement $\Delta x(t)$ and the phase variation $\Delta \theta(t)$ is:

$$
\Delta \theta(t)=\frac{4 \pi \Delta x(t)}{\lambda}
$$

where $\lambda$ is the wavelength of the transmitted signal.

\section{System Description}

The first version consists of using a VNA and one antenna where the $S_{11}$ parameter is considered. The second version consists of using a VNA and two antennas where the $\mathrm{S}_{21}$ parameter is considered. Measurements were performed while holding the breath for duration of 15 seconds. This duration may vary in the order of milliseconds in order to sustain synchronization between the VNA and the ECG. The number of points taken for the signals measured with the VNA is 20000. The use of a VNA accommodates a quick and simple installation process. Fig. 1 represents the proposed system. Beside simplicity, many benefits are offered upon the use of a VNA (N5230A); these include the following features:

$$
\begin{aligned}
& \text { - Full 4-port S-parameter and balanced } \\
& \text { measurements up to } 20 \mathrm{GHz} \\
& \text { - } 120 \mathrm{~dB} \text { dynamic range at } 20 \mathrm{GHz} \\
& \text { - Trace noise }<0.006 \mathrm{~dB} \text { at } 100 \mathrm{kHz} \text { IFBW } \\
& \text { - Sampling period }<4 \mu \mathrm{s} \\
& \text { - Automatic port extension automatically corrects in } \\
& \text { fixture measurements. } \\
& \text { - Advanced connectivity with LAN, USB, and GPIB } \\
& \text { interfaces }
\end{aligned}
$$

One of the most powerful features gained using a VNA is the possibility of tuning both the operational frequency and the transmitted power. In addition, a VNA allows controlling different parameters such as the sweep time and the number of points for a specific trace; thus the sampling rate can be pre-determined.

Two wide band antennas (Q-par Angus Ltd) were used in experiments performed between 2 and $18 \mathrm{GHz}$. The Qpar Angus Ltd (model number WBH2-18HN/S) has the following specifications:

-Frequency: $2-18 \mathrm{GHz}$

- Nominal gain: $10-22 \mathrm{dBi}$

- Nominal beamwidth: $6-11$ degrees

-VSWR: $<2.5: 1$ (typically $<2.0: 1$ )

- Cross polar: $<-17 \mathrm{~dB}$

- Power handling: SMA 50 Watts CW, type N 80 Watts CW

- Construction: metal/glass-resin composite

-Dimensions: 622*165*165 mm approx.

- Connector: SMA or type N

-Weight: $2.7 \mathrm{~kg}$, temperature: from $-40{ }^{\circ} \mathrm{C}$ to $70{ }^{\circ} \mathrm{C}$

The operational frequency used in these measurements is $16 \mathrm{GHz}$. The choice of this frequency is made according to two reasons. The first one is to obtain higher phase variation as it is directly proportional to the frequency, and the second reason is due to the equipment's limits $(2-16$ $\mathrm{GHz}$ for the antennas). An extension of the system operating at $60 \mathrm{GHz}$ is described in [16, 17]. The measurements procedure starts by sending a continuous wave signal at the desired frequency. The signal is generated by the VNA and driven to the transmitting antenna, which is directed toward the person's chest. The transmitted signal is reflected off the person chest and received by an antenna similar to the transmitter. The received signal is driven to the VNA where the phase variation of $S_{21}$ is computed. This phase corresponds to the difference between the phase of the received signal and the phase of the transmitted signal. The variation of the phase of $S_{21}$ is due to the chest displacement. Hence, it contains information about the cardiopulmonary signals when breathing normally, and about the heartbeat signal when holding the breath. The same procedure is used when the phase of $S_{11}$ is measured. In this case, only one antenna is used for both transmission and reception.

\section{Smoothing Technique}

Since the heartbeat rate is based on the detection of the signal peaks, a smoothing method is applied in order to reduce the number of surrounding peaks. The smoothing method is based on the Newton relation:

$$
(a+b)^{2 m}=\sum_{k=0}^{2 m} C_{2 m}^{k} a^{k} b^{2 m-k}
$$

where $n=2 m+1$ is the length of the smoothing window and

$$
C_{n}^{k}=\frac{n !}{k !(n-k) !}, \mathrm{k}, \mathrm{n} \in \mathbb{N}, 0 \leq \mathrm{k} \leq \mathrm{n}
$$

Consider the obtained phase variations represented by a 
vector $P$ of $N$ samples:

$$
P=[p(1) \ldots p(i-m) \ldots p(i) \ldots p(i+m) \ldots p(N)]
$$

The smoothing technique replaces a given point $p(i)$ by the weighted average of its surrounding points. This is obtained according to the following relation:

$$
p_{s}(i)=\frac{1}{2^{2 m}} \sum_{k=0}^{2 m} C_{2 m}^{k} p(i-m+k)
$$

where $i$ should verify the following condition:

$$
m+1 \leq i \leq N-m
$$

In general, the length of the smoothing window is chosen according to the following criterion:

$$
1 \% \leq \frac{n}{N} \leq 20 \%
$$

On the other hand, the length of the smoothing window should be decreased accordingly when the corresponding point $p(i)$ is near either sides of the vector $P$. For example, when $1 \leq i \leq m, p(i)$ is replaced by:

$$
p_{S}(i)=\frac{1}{4^{i-1}} \sum_{k=0}^{2(i-1)} C_{2(i-1)}^{k} p(k+1)
$$

When $N-m+1 \leq i \leq N, p(i)$ is replaced by:

$$
p_{s}(i)=\frac{1}{4^{N-i}} \sum_{k=0}^{2(N-i)} c_{2(N-i)}^{k} p(2 i-N+k)
$$

\section{HeARtbeat SignAl Detection}

This section presents the results obtained upon the detection of the heart activity using two different schemes. The first scheme is based on measuring the phase of $S_{11}$ where single antenna is used for both transmitting and receiving. The second scheme is based on measuring the phase of $S_{21}$ where two antennas are used to respectively transmit and receive signals.

\section{One Antenna}

Measurements are performed for several transmitted powers between 0 and $-25 \mathrm{dBm}$, and for a distance of $1 \mathrm{~m}$ to the person. Fig. 2 (a) shows the heartbeat signal detected using an ECG, while Fig. 2 (b) shows the phase variation of $\mathrm{S}_{11}$ due to the heartbeat activity. These two signals are obtained simultaneously for the same patient. Fig. 2 (c) shows the heartbeat signal after applying the previously described smoothing technique.

Beside that the heartbeat signal detected with an ECG represents the electrical activity of the heart, the goal of the microwave Doppler system is to measure the heartbeat rate; this corresponds to the average of the R-R intervals of the ECG signal.

Fig. 3 shows the heartbeat signals that correspond to the phase of $S_{11}$ detected by the proposed microwave system for several power levels of the transmitted signal: $0 \mathrm{dBm}$ (a), $-5 \mathrm{dBm}(\mathrm{b}),-10 \mathrm{dBm}(\mathrm{c}),-15 \mathrm{dBm}(\mathrm{d}),-20 \mathrm{dBm}(\mathrm{e})$, and $-25 \mathrm{dBm}(\mathrm{f})$. It can be noticed that as the power decreases, the signal-to-noise ratio (SNR) decreases. However, the peaks of the signals can still be detected. The effect of smoothing these signals is shown in Fig. 4.

\section{Two Antennas}

Measurements are performed for several transmitted power values. The phase variation of $S_{21}$ is measured. Fig. 5 (a) shows the heartbeat signal detected using an ECG, while Fig. 5 (b) shows the phase variation of $S_{21}$ due to heartbeats detected using a transmitted power of $-5 \mathrm{dBm}$. These two signals are obtained simultaneously for the same patient. Fig. 5 (c) shows the heartbeat signal (the phase of $\mathrm{S}_{21}$ ) after applying a smoothing technique.

Fig. 6 shows the phase variation of $S_{21}$ due to the heartbeats detected by the proposed microwave system for several transmitted powers: $0 \mathrm{dBm}(\mathrm{a}),-5 \mathrm{dBm}(\mathrm{b}),-10$ $\mathrm{dBm}(\mathrm{c}),-15 \mathrm{dBm}(\mathrm{d}),-20 \mathrm{dBm}$ (e), and $-25 \mathrm{dBm}(\mathrm{f})$. As for the phase variation of $S_{11}$, the signal-to-noise ratio (SNR) decreases as the power of the transmitted signal decreases. The peaks of the signals can still be detected even for low power levels of the transmitted signal. The effect of smoothing these signals is shown in Fig. 7.

Note that y-axis of Figs. 2 (b, c) and Figs. 5 (b, c) represent the phase in degrees while the y-axis of Fig. 2 (a) and Fig. 5 (a) stand for the ECG signal represented by 8 bits digital values.

\section{$\mathrm{S}_{11}$ Versus $_{21}$ : Heartbeat Rate and Heart Rate VARIABILITY}

Related works tend to extract the average heartbeat rate for a specific window. This does not provide information about the time-variation of the HR, i.e. the HRV. Thus, a peak detection technique is required to track the peaks of the signal. In this work, the heartbeat rate and the HRV of the signals detected for different power levels are extracted. Values obtained from both parameters $S_{11}$ and $S_{21}$, for original and smoothed signals, are compared to HR and the HRV obtained from the ECG reference signal.

The Peak Detection Method (PDM) used in this work is based on the following steps:

1. the maximum value of a 2-seconds window starting at time $=0$ is considered as the first peak

2. the window is shifted to the right by $n$ points and another maximum value is searched

3. the new maximum value is conserved if it satisfies two conditions: the first one is to remain maximum for a duration of 0.5 seconds ( 0.25 at right and 0.25 at left), and the second one is to have a value greater than a threshold $T$

The size of the window is chosen as the minimum heartbeat rate is 30 beats per minute, hence the maximum duration between 2 beats is 2 seconds. $n$ is chosen according to the number of points to be read at time. In this code $n$ is equal to 10 . The value of $T$ is chosen as a mean 
between the average value and the maximum value of the signal.

Fig. 8 shows the HR relative error of the Doppler radar system, for the phases of both $S_{11}$ and $S_{21}$, with respect to the reference ECG signal. The effects of smoothing the signal are also shown in this figure.

It can be noticed that both $\mathrm{S}_{11}$ and $\mathrm{S}_{21}$ parameters' can detect the heartbeat signals with relative errors around $1.5 \%$ at $0 \mathrm{dBm}$. However, using the phase of $\mathrm{S}_{11}$ shows a relative error for the HR error greater than $10 \%$ when the power of the transmitted signal becomes less than -10 $\mathrm{dBm}$. On the other hand, $\mathrm{S}_{21}$ shows the possibility of extracting the HR even at $-25 \mathrm{dBm}$, with a relative error around $4 \%$. The effect of smoothing the signals is higher for $S_{11}$ than for $S_{21}$; this is due to the lower SNR obtained for $S_{11}$. Specifically, the HR relative error for $S_{11}$ changes between $1.5 \%$ and $26 \%$ for transmitted power between 0 and $-25 \mathrm{dBm}$ respectively. The smoothing technique changes these values to $2 \%$ and $12 \%$ respectively. Higher accuracy is obtained when measuring the phase of $\mathrm{S}_{21}$ where HR relative error between 0 and $3.5 \%$ is obtained. Smoothing the signal decreases this relative error to $1.5 \%$.

The HRV is a measure of the beat-to-beat variations (R$\mathrm{R}$ interval for the ECG signal). HRV is regarded as an activity indicator of autonomic regulation of circulatory function; notice that controversy exists over whether this is an accurate metric for analyzing cardiovascular autonomic control [18-20]. The extraction of the heartbeat rate requires the use of simple averaging over a specific window. However, this lacks providing the time-variation of the heartbeat rate. On the other hand, the extraction of the heart rate variability requires peak-to-peak detection. Hence, a PDM is used. This technique is based on tracking the maximum value in a sliding window, which is greater than a specific threshold. The size of the window is 2 seconds starting at the last peak detected in the preceding window. The minimum and maximum durations between two consecutive peaks are 0.5 and 1.5 seconds respectively. These values correspond to a heartbeat rate between 120 and 40 beats per minute.

It can be noticed that higher accuracy is obtained when using two antennas system, while using one antenna system requires higher directivity and positioning of the antenna.

Fig. 9 shows the HRV relative error of the Doppler radar system, for the phases of both $S_{11}$ and $S_{21}$ parameters, with respect to the reference ECG signal. The effects of smoothing the signal are shown as well. The proposed system shows the possibility of detecting the peaks of the signal with high accuracy. The HRV relative error for $S_{11}$ changes between $2.5 \%$ and $28 \%$ for transmitted power between 0 and $-25 \mathrm{dBm}$. The HRV relative error for $\mathrm{S}_{21}$ changes between $1.5 \%$ and $21 \%$. On the other hand, smoothing the signal changes the HRV error as follows: 3 $-11 \%$ interval for $S_{11}$, and $1-6 \%$ for $S_{21}$.

\section{CONCLUSION}

A microwave system is proposed in order to detect the heart beatings of a person from a distance of 1 meter. A comparison between two versions of the proposed system is presented. The phase variations of parameters $S_{11}$ and $S_{21}$ obtained with a VNA due to the heart activity are extracted and compared to a simultaneous ECG signal. Both schemes, with one and two antennas, show the possibility of detecting the heartbeat activity and allow extracting the heartbeat rate and the heart rate variability. Measurements are performed at $16 \mathrm{GHz}$ and for several transmitted power levels between 0 and $-25 \mathrm{dBm}$. Beside its installation simplicity, the VNA provides the opportunity of testing several transmitted powers. Determining the minimum possible transmitted power would be safer for the patient and the medical staff.

Using one antenna scheme shows the possibility of extracting the heartbeat rate, while it lacks determining the heart rate variability at low power levels of the transmitted signal. This is due to the sensitive positioning of the antenna toward the chest of the person. On the other hand, the two antennas scheme shows higher accuracy in extracting both HR and HRV. A smoothing technique is applied to the signal where high accuracy is observed in extracting the HR and the HRV for both signals obtained with $\mathrm{S}_{11}$ and $\mathrm{S}_{21}$.

Future work will focus on performing measurements for several operational frequencies simultaneously with an ECG. This helps determining the optimum operational frequency for the minimum transmitted power.

\section{REFERENCES}

[1] H.-R. Chuang, Y. Chen, and K.-M. Chen, "Automatic cluttercanceller for microwave life-detection systems," IEEE Transactions on Instrumentation and Measurement, vol. 40, no. 4, pp. 747 -750, Aug. 1991.

[2] J. C. Lin, "Microwave sensing of physiological movement and volume change: A review," Bioelectromagnetics (1992), vol. 13, pp. $557-565$.

[3] A. De Groote, M. Wantier, G. Cheron, M. Estenne, and M. Paiva, "Chest wall motion during tidal breathing," Journal of Applied Physiology (1997), vol. 83, no. 5, pp. 1531-1537.

[4] G. Ramachandran and M. Singh, "Three-dimensional reconstruction of cardiac displacement patterns on the chest wall during the $\mathrm{P}$, QRS, and T-segments of the ECG by laser speckle interferometry," Medical and Biological Engineering and Computing (1989), vol. 27, no. 5, pp. 525-530.

[5] F. Mohammad-Zahed, F. Taghibakhsh and B. Kaminska, "Contactless Heart Monitoring," 2007 IEEE, Session 33: Biomedical Signal Processing-I, pp. 583-585.

[6] K. M. Chen, Y. Huang, J. Zhang, and A. Norman, "Microwave lifedetection systems for searching human subjects under earthquake rubble and behind barrier", IEEE Trans. Biomed. Eng. Jan. 2000, vol. 47 , no. 1 , pp. $105-114$.

[7] A. D. Droitcour, V. M. Lubecke, J. Lin, and O. Boric-Lubecke, "A microwave radio for Doppler radar sensing of vital signs," in IEEE MTT-S Int. Microw. Symp. Dig., May 2001, pp. 175-178.

[8] A. D. Droitcour, O. Boric-Lubecke, V. M. Lubecke, and J. Lin, "0.25 $\mu \mathrm{m}$ CMOS and BiCMOS single chip direct conversion Doppler radars for remote sensing of vital signs," in IEEE Int. SolidState Circuits Conf. Tech. Dig., Feb. 2002, pp. 348-349. 
[9] O. Boric-Lubecke, G. Awater, and V. M. Lubecke, "Wireless LAN PC Card Sensing of Vital Signs," IEEE Topical Conference on Wireless Communication Technology (2003), pp. 206-207.

[10] Y. Xiao, J. Lin, O. Boric-Lubecke, and V. M. Lubecke, "A Ka-band low power Doppler radar system for remote detection of cardiopulmonary motion," presented at the 27th IEEE Annu. Eng. Med. Biol. Soc. Conf., Sep. 1-4, 2005.

[11] C. Gu, C. Li, J. Lin, J. Long, J. Huangfu, and L. Ran, "InstrumentBased Noncontact Doppler Radar Vital Sign Detection System Using Heterodyne Digital Quadrature Demodulation Architecture," IEEE Transactions on Instrumentation and Measurement, Vol. 59, No. 6, pp. 1580-1588, June 2010.

[12] V. M. Lubecke, O. B. Lubecke, A. H. Madsen, and A. E. Fathy, "Through-the-wall radar life detection and monitoring," in IEEE International Microwave Symposium, 2007, pp. 769-772.

[13] C. Li and J. Lin, "Random body movement cancellation in doppler radar vital sign detection," IEEE Trans. on Microw. Theory Techn., vol. 56, pp. 3143-3152, 2008.

[14] I. V. Mikhelson, S. Bakhtiari, T. W. Elmer, and A. V. Sahakian, "Remote sensing of heart rate and patterns of respiration on a stationary subject using $94-\mathrm{GHz}$ millimeter-wave interferometry," IEEE Transactions on Biomedical Engineering, Vol. 58, Issue 6, pp. $1671-1677,2011$
[15] A. A. Serra, P. Nepa, G. Manara, G. Corsini, and J. L. Volakis, "A single on-body as a sensor for cardiopulmonary monitoring," IEEE Antennas and Wireless Propagation Letters, Vol. 9, pp. 930-933, 2010.

[16] D. Obeid, S. Sadek, G. Zaharia, and G. El Zein, "Non-Contact Heartbeat Detection at 2.4, 5.8 and $60 \mathrm{GHz}$ : A Comparative Study," Microwave Opt. Technol. Lett., vol. 51, no. 3, pp. 666-669, March 2009.

[17] D. Obeid, S. Sadek, G. Zaharia, and G. El Zein, "Multitunable Microwave System for Touchless Heartbeat Detection and Heart Rate Variability Extraction," Microwave Opt. Technol. Lett., vol. 52, no. 1, pp. 192-198, Jan. 2010

[18] P. Joseph, U. R. Acharya, C. K. Poo, J. Chee, L. C. Min, S. S. Iyengar, and $\mathrm{H}$. Wei, "Effect of reflexological stimulation on heart rate variability," ITBM-RBM, vol. 25, pp. 40-45, 2004.

[19] A. Al-Hazimi, N. Al-Ama, A. Syiamic, R. Qosti, and K. AbdelGalil, "Time-domain analysis of heart rate variability in diabetic patients with and with- out autonomic neuropathy," Annals of Saudi Medicine, vol. 22, no. Nos 5-6, pp. 400-403, 2002.

[20] D. C. Lin and R. L. Hughson, "Modeling heart rate variability in healthy humans: A turbulence analogy," Physical Review Letters, vol. 86(8), pp. 1650-1653, 2001.

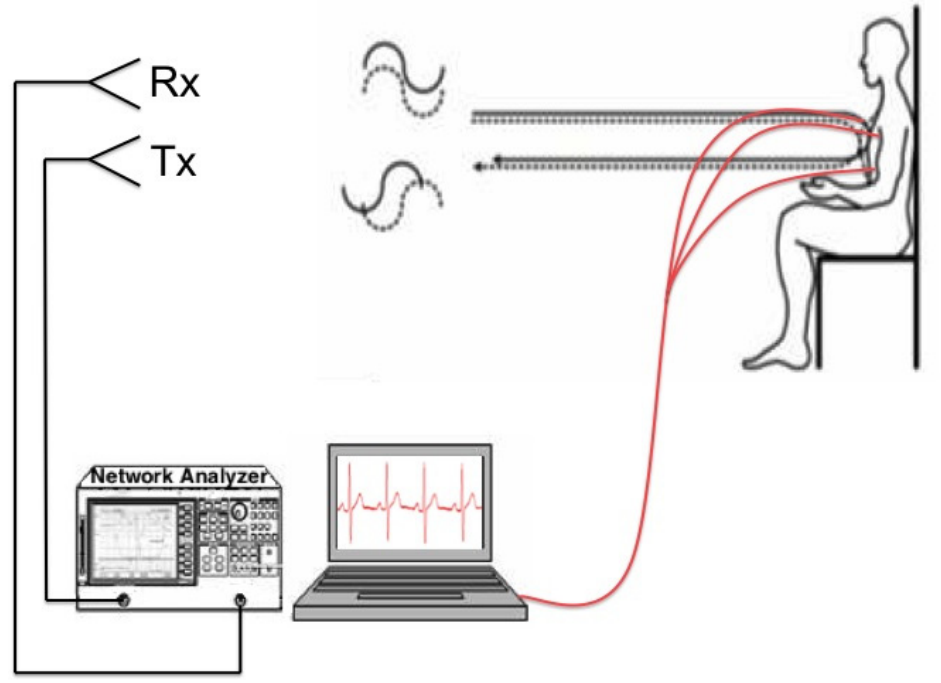

Fig. 1. System design: Pc-based ECG and VNA (only the Tx antenna is used for $\mathrm{S}_{11}$ ). 

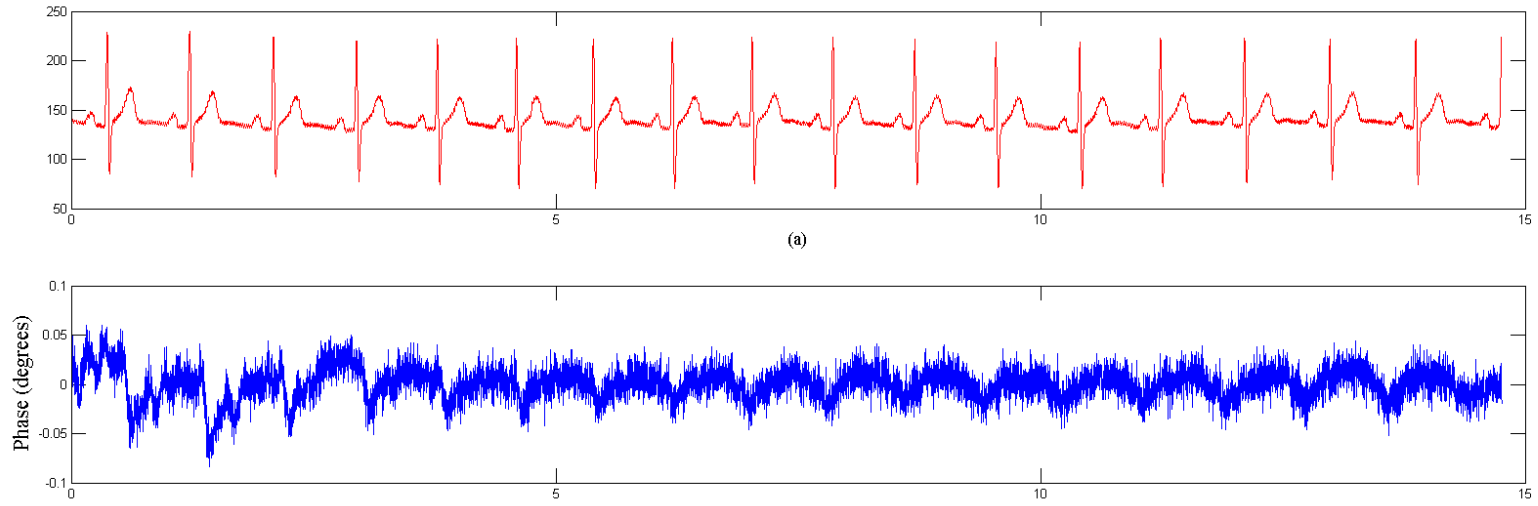

(b)

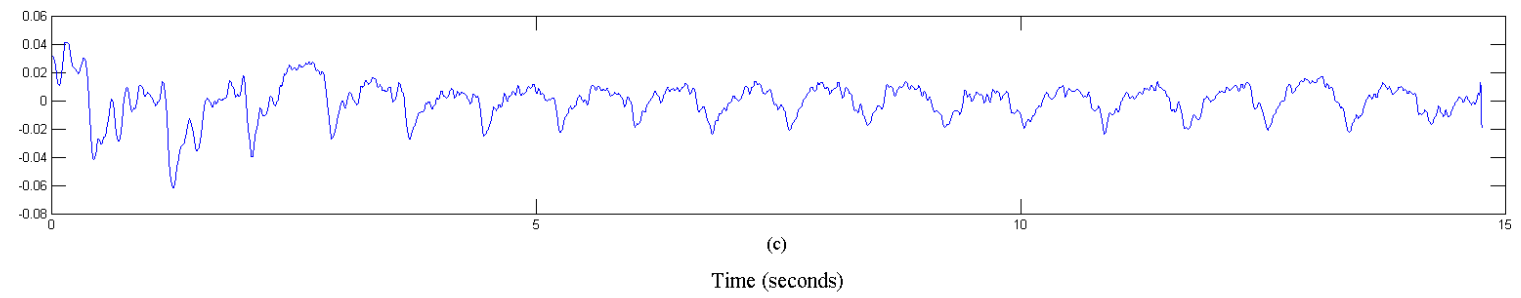

Fig. 2. ECG signal (a) and phase variations of $S_{11}$ due to heartbeats detected at $16 \mathrm{GHz}$ and $-5 \mathrm{dBm}$ : original signal (b), smoothed signal (c).

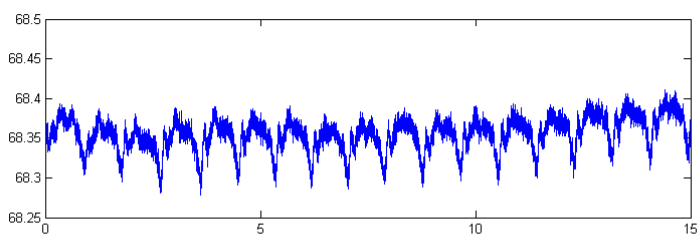

(a)

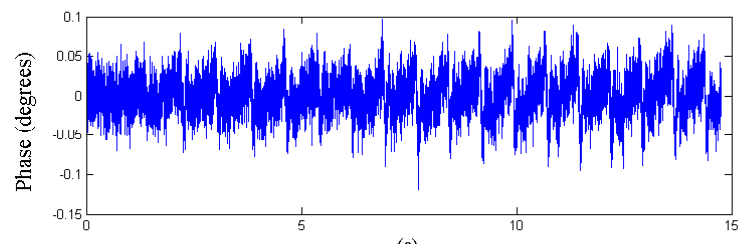

(c)

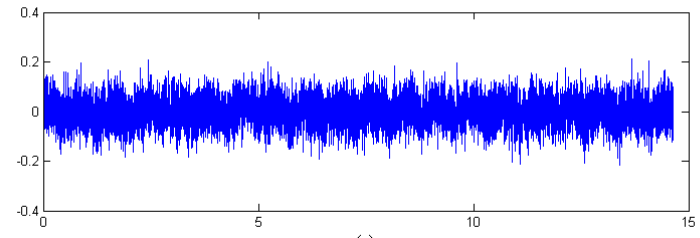

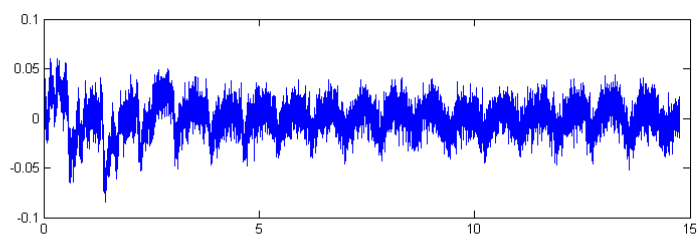

(b)

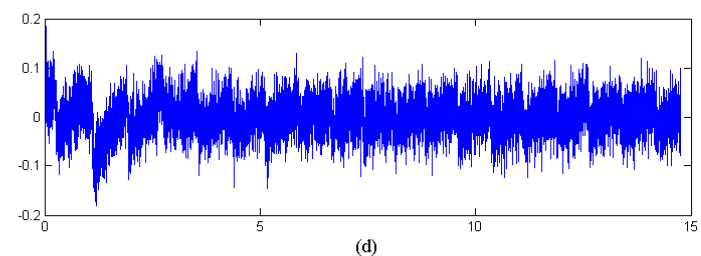

(d)

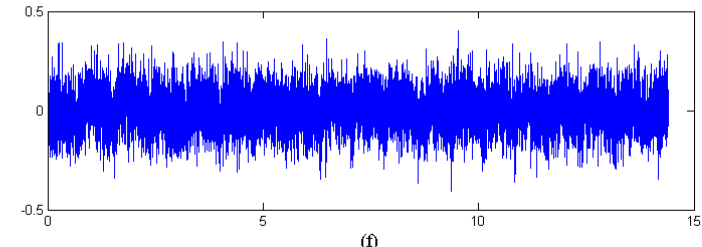

(f)

Time (seconds)

Fig. 3. Phase variations of $S_{11}$ due to heartbeats detected at $16 \mathrm{GHz}$ for $0 \mathrm{dBm}(\mathrm{a}),-5 \mathrm{dBm}(\mathrm{b}),-10 \mathrm{dBm}(\mathrm{c}),-15 \mathrm{dBm}(\mathrm{d}),-20 \mathrm{dBm}$ (e), and $-25 \mathrm{dBm}$ (f). 

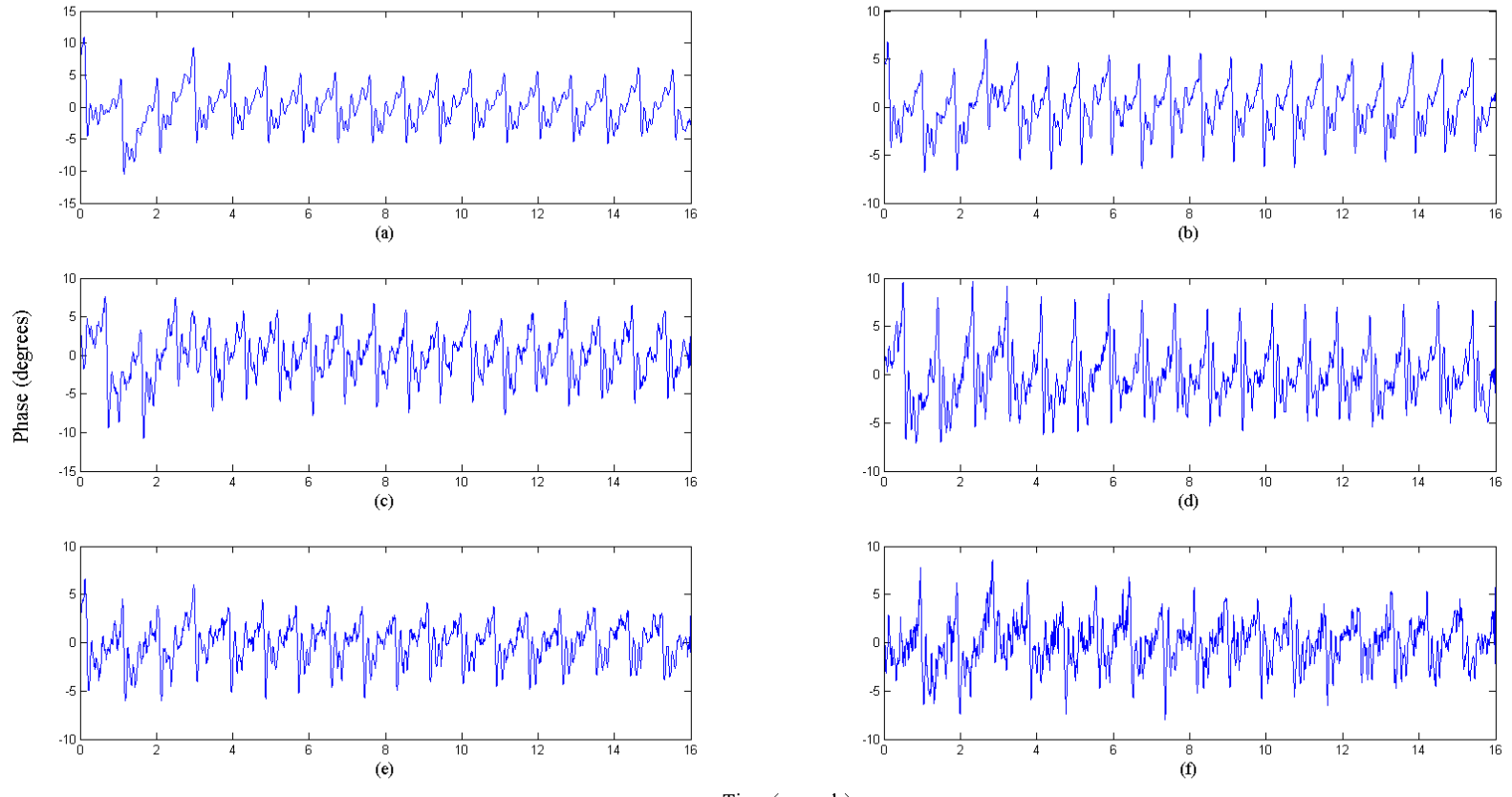

Fig. 4. Phase variations of $S_{11}$, after applying the smoothing technique, due to heartbeats detected at $16 \mathrm{GHz}$ for $0 \mathrm{dBm}(\mathrm{a}),-5 \mathrm{dBm}(\mathrm{b}),-10 \mathrm{dBm}(\mathrm{c}),-15 \mathrm{dBm}$ (d), $-20 \mathrm{dBm}(\mathrm{e})$, and $-25 \mathrm{dBm}(\mathrm{f})$.
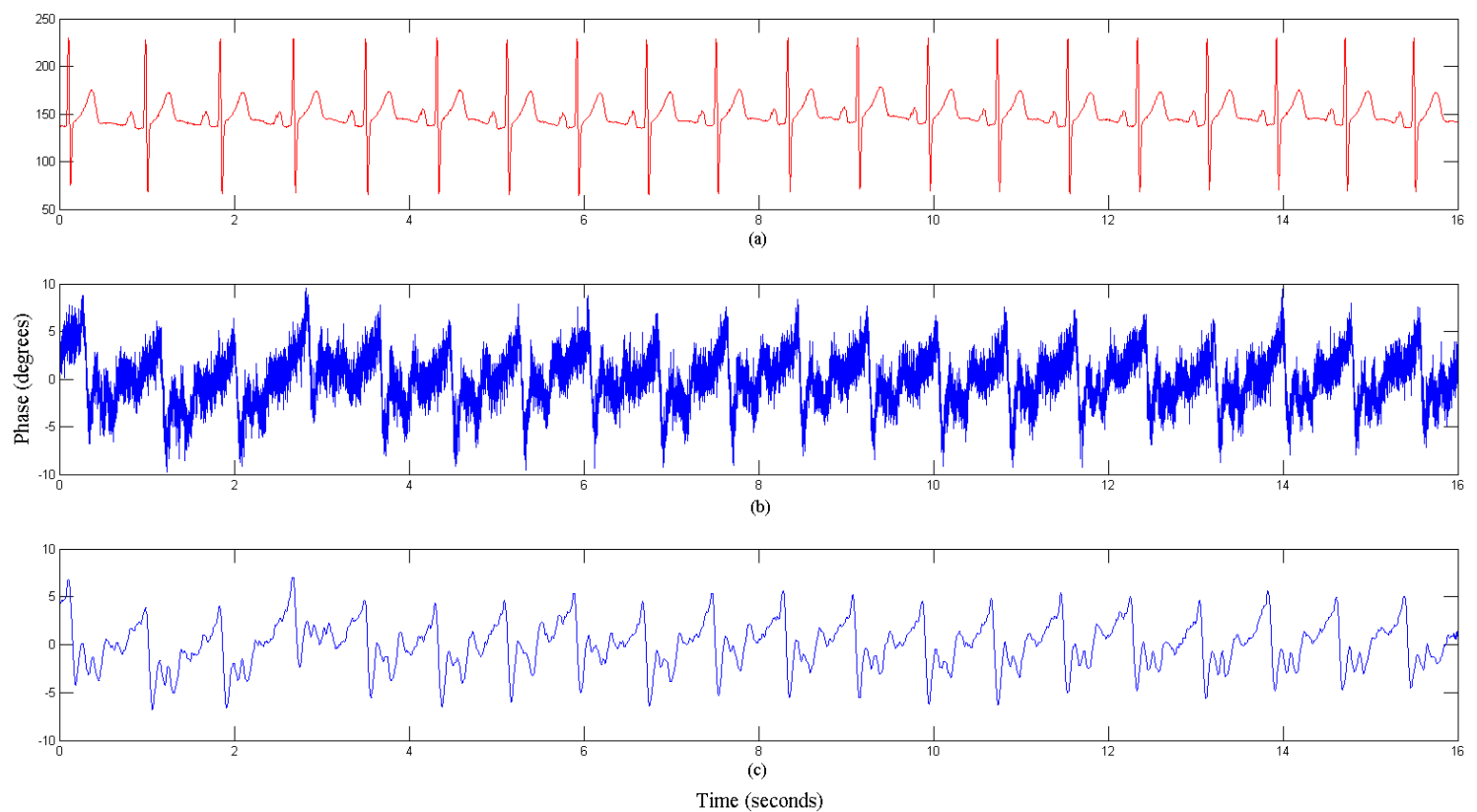

Fig. 5. ECG signal (a) and phase variations of $\mathrm{S}_{21}$ due to heartbeats detected at $16 \mathrm{GHz}$ and $-5 \mathrm{dBm}$ : original signal (b), smoothed signal (c). 

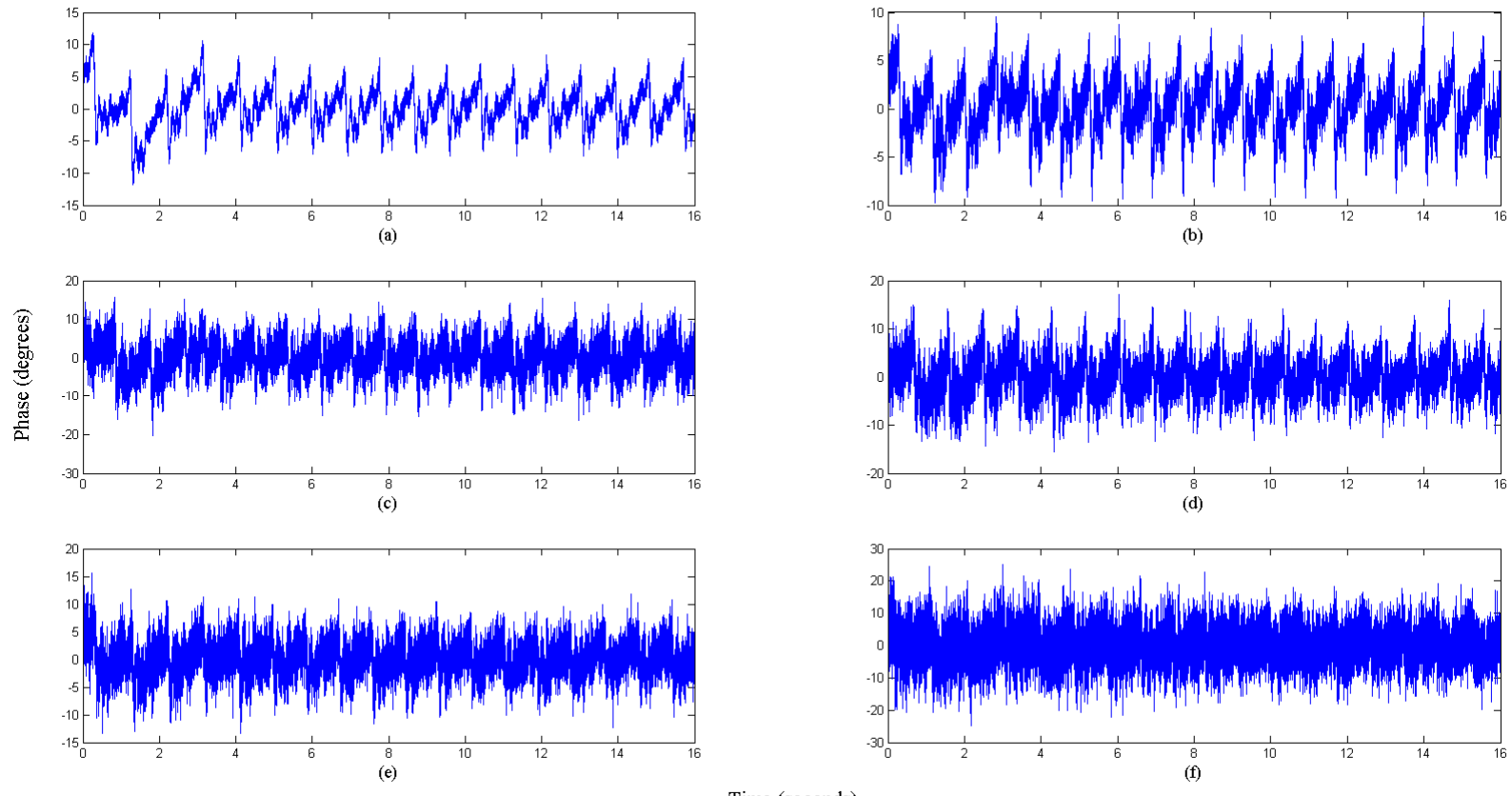

Fig. 6. Phase variations of $S_{21}$ due to heartbeats detected at $16 \mathrm{GHz}$ for $0 \mathrm{dBm}(\mathrm{a}),-5 \mathrm{dBm}(\mathrm{b}),-10 \mathrm{dBm}(\mathrm{c}),-15 \mathrm{dBm}(\mathrm{d}),-20 \mathrm{dBm}(\mathrm{e})$, and $-25 \mathrm{dBm}(\mathrm{f})$.
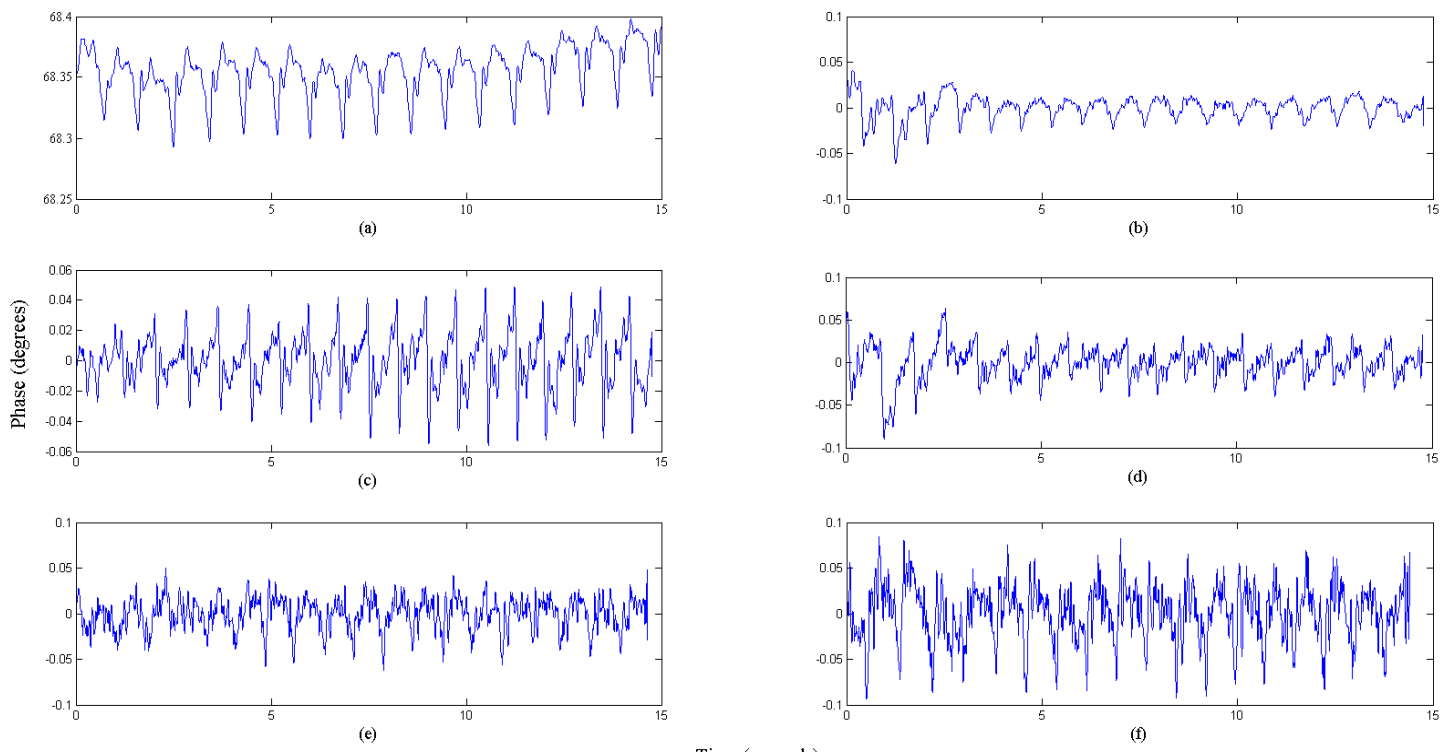

Fig. 7. Phase variations of $\mathrm{S}_{21}$, after applying the smoothing technique, due to heartbeats detected at $16 \mathrm{GHz}$ for $0 \mathrm{dBm}(\mathrm{a}),-5 \mathrm{dBm}(\mathrm{b}),-10 \mathrm{dBm}(\mathrm{c}),-15$ $\mathrm{dBm}(\mathrm{d}),-20 \mathrm{dBm}(\mathrm{e})$, and $-25 \mathrm{dBm}(\mathrm{f})$. 


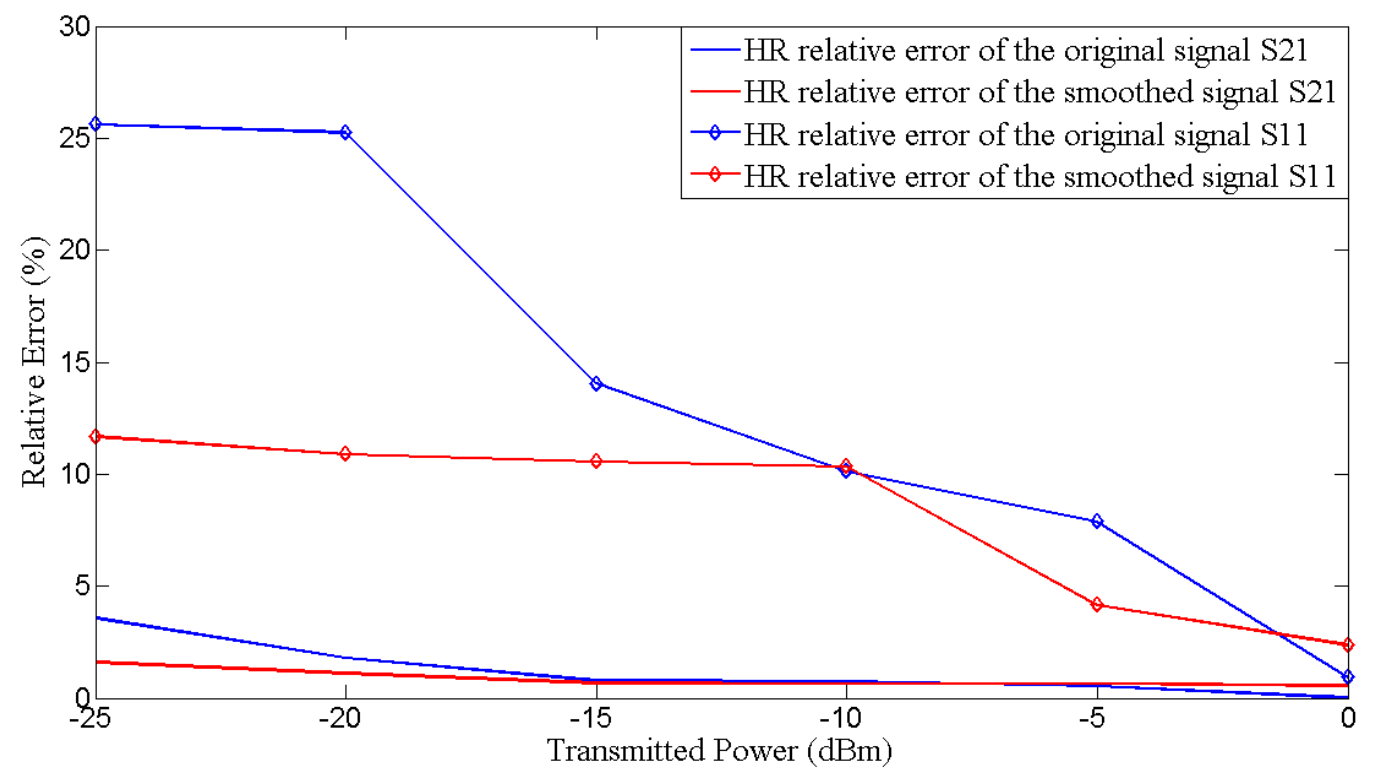

Fig. 8. HR relative error of $S_{11}$ and $S_{21}$ for both original and smoothed signals.

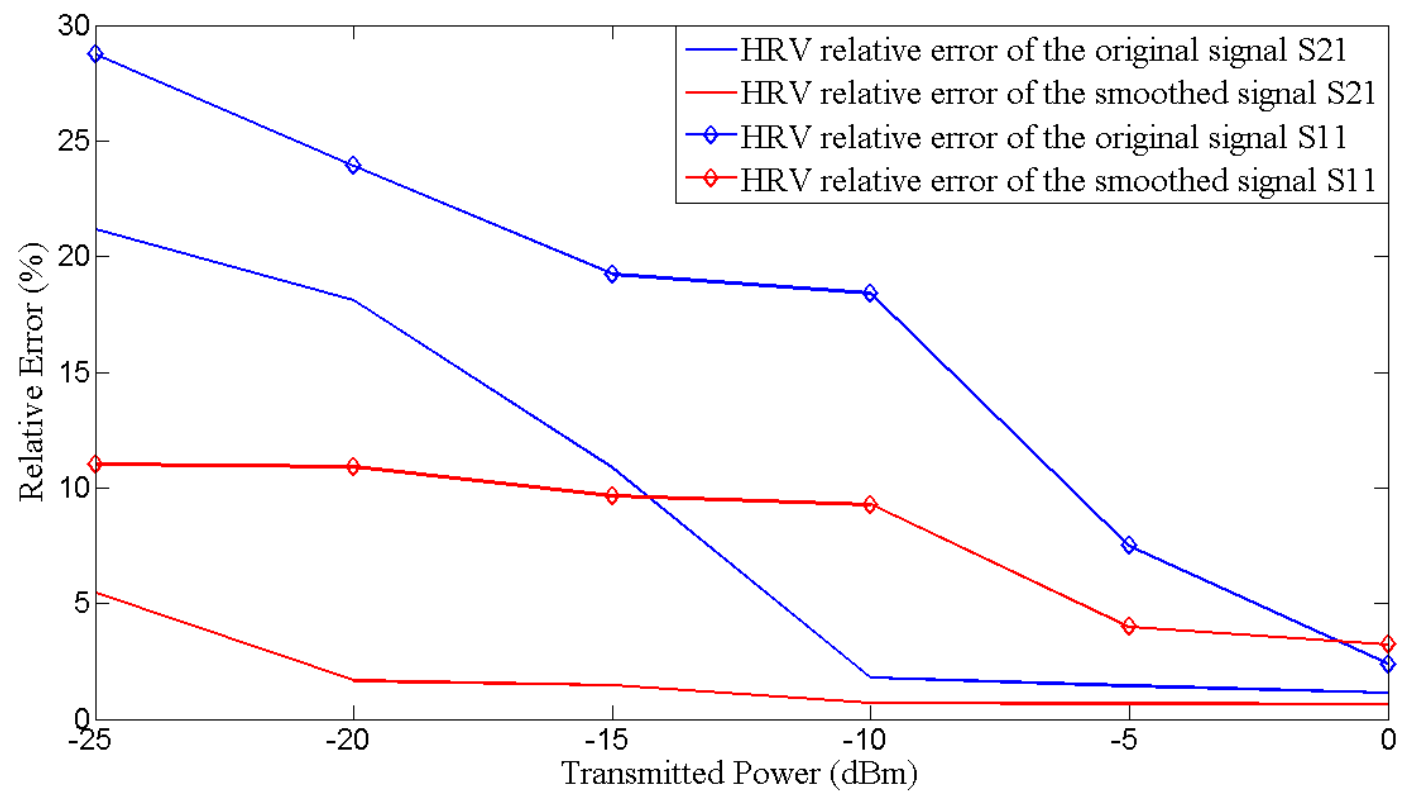

Fig. 9. HRV relative error of $S_{11}$ and $S_{21}$ for both original and smoothed signals. 